\title{
Florística de trepadeiras numa floresta estacional semidecídua, Rio Claro - Araras, Estado de São Paulo, Brasil
}

\author{
RENATA G. UDULUTSCH${ }^{1}$, MARCO A. ASSIS ${ }^{1,2}$ e DOUGLAS G. PICCHI ${ }^{1}$
}

(recebido: 7 de agosto de 2002; aceito: 20 de novembro de 2003)

\begin{abstract}
Floristic of climbers in a seasonal semi-deciduous forest, Rio Claro - Araras, State of São Paulo, Brazil). This study aimed at characterizing the composition of climbers within a 230 ha fragment in Seasonal Semi-deciduous Forest located between the municipalities of Rio Claro and Araras ( $22^{\circ} 21^{\prime} \mathrm{S}$ and $47^{\circ} 28^{\prime}-47^{\circ} 29^{\prime} \mathrm{W}, 630 \mathrm{~m}$ of altitude). Samples were collected monthly from September/2000 to April/2002, and the climbers were classified into two different groups (herbaceous vines or woody lianas); and according to their climbing patterns they were divided into twines, with tendrils, or scandent. A total of 148 species, distributed in 82 genera and 33 families were identified. Bignoniaceae (29 species), Asteraceae (19), Sapindaceae (12), Malpighiaceae (11), and Convolvulaceae (nine) were the richest families, with $54 \%$ of total species. Bignoniaceae presented the greatest number of species, and genera (16) confirming reports that it is the richest climbers family in the Seasonal Semideciduous Forest, as it has previously been stated by studies carried out in lowland neotropical forests. Like in other studies carried out in Seasonal Semi-deciduous Forests, woody climbers stood for approximately 2/3 of the diversity of all climbers. It was possible to record the predominance of both twining species $(43 \%)$ and those with tendrils $(39 \%)$ over the scandent species (18\%). Although the composition of species may be quite distinct, the richest families and genera identified in this study are often identified as having the same relevance in previous studies conducted in Seasonal Semi-deciduous Forest in the state of São Paulo.
\end{abstract}

Key words - climbers, floristic, lianas, semi-deciduous forest, tropical forest

RESUMO - (Florística de trepadeiras numa floresta estacional semidecídua, Rio Claro - Araras, Estado de São Paulo, Brasil). Visando caracterizar a composição florística de trepadeiras nas florestas estacionais semidecíduas, foi realizado o levantamento das espécies num fragmento de 230 ha, abrangido pelos municípios de Rio Claro e Araras $\left(22^{\circ} 21^{\prime}\right.$ 'S e $47^{\circ} 28^{\prime}-47^{\circ} 29^{\prime} \mathrm{W}, 630 \mathrm{~m}$ de altitude). As coletas foram mensais durante o período de setembro de 2000 a abril de 2002, sendo as trepadeiras classificadas como herbáceas ou lenhosas e quanto às suas formas de escalar; em volúveis, com gavinhas ou não preensoras. Foram encontradas 148 espécies, distribuídas em 82 gêneros e 33 famílias. Bignoniaceae (29 espécies), Asteraceae (19), Sapindaceae (12), Malpighiaceae (11) e Convolvulaceae (nove) foram as famílias mais ricas, abrangendo $54 \%$ das espécies amostradas. Bignoniaceae apresentou o maior número de espécies e gêneros (16), corroborando outros relatos de que é a família mais rica em trepadeiras nas florestas estacionais semidecíduas e na maioria dos estudos realizados em florestas neotropicais de baixas altitudes. Como em outros estudos realizados em florestas estacionais semidecíduas, as trepadeiras lenhosas representaram aproximadamente $2 / 3$ da riqueza desse componente. Verificou-se a prevalência das espécies volúveis (43\%) e das espécies dotadas de gavinhas $(39 \%)$ em relação às espécies não preensoras $(18 \%)$. Se, por um lado, a composição de espécies se mostrou bastante distinta em relação a outros estudos conduzidos em florestas estacionais semidecíduas no Estado de São Paulo, as famílias e gêneros mais ricos em espécies são, em grande parte, concordantes.

Palavras-chave - floresta semidecídua, floresta tropical, florística, lianas, trepadeiras

\section{Introdução}

Nas últimas décadas, a realização de inúmeros trabalhos tem propiciado um considerável aumento do conhecimento sobre as florestas estacionais semidecíduas, uma extensa e importante formação florestal brasileira extra-amazônica (Pagano \& Leitão Filho 1987, Pagano et al. 1987, Salis et al. 1995, Torres et al. 1997, Oliveira Filho \& Fontes 2000).

\footnotetext{
1. Universidade Estadual Paulista, Instituto de Biociências, Departamento de Botânica, Caixa Postal 199, 13506-900 Rio Claro, SP, Brasil.

2. Autor para correspondência: massis@rc.unesp.br
}

Entretanto, a maioria dos estudos realizados apresentou enfoque específico para o componente arbóreo, em detrimento dos demais componentes florísticos. Entre os componentes pouco estudados, o das trepadeiras é composto de um grupo de plantas bastante característico dessas florestas, tendo um marcante papel na dinâmica dessas comunidades, tanto no que se refere às relações com as plantas que as sustentam (forófitos) quanto no fornecimento de recursos à fauna (Morellato \& Leitão Filho 1996, Engel et al. 1998).

De acordo com Veloso (1991), trepadeiras são plantas herbáceas ou lenhosas que vivem apoiadas em 
outra planta ou substrato, cujas gemas acima do solo são protegidas por catáfilos. Trepadeiras, portanto, são plantas cujo crescimento em altura depende da sustentação mecânica fornecida por outras plantas (Putz \& Windsor 1987). Enquanto as árvores investem recursos em tecidos de sustentação, trepadeiras investem no crescimento rápido em altura. As trepadeiras crescem geralmente em direção ao dossel, sombreando as árvores que as sustentam e competindo com estas por luz, água e nutrientes (Clark \& Clark 1990, Gentry 1991). Com isso, as taxas de crescimento e mortalidade das árvores são alteradas (Putz \& Chai 1987), o que pode atuar como uma importante força seletiva na evolução das árvores em matas tropicais (Putz 1984).

A maior diversidade e abundância de trepadeiras ocorre em florestas tropicais, onde são elementos característicos (Richard 1952, Peñalosa 1984, Putz 1984, Gentry 1991). Por esse motivo, essas plantas constituem um importante componente florístico, estrutural e funcional nessas florestas (Gentry 1991, Engel et al. 1998).

Apesar de sua importância nas florestas tropicais, raramente as trepadeiras são tidas como objeto principal nos estudos florísticos, sendo, muitas vezes, coletadas apenas casualmente (Gentry 1991, Morellato \& Leitão Filho 1998). No Brasil, os poucos estudos florísticos que enfocaram mais especificamente as trepadeiras são recentes, tendo sido realizados na vegetação amazônica (Gentry 1978, Prance 1994, Ribeiro et al. 1999), em floresta atlântica (Lima et al. 1997) e em floresta estacional semidecídua da região sudeste (Morellato \& Leitão Filho 1996, 1998, Hora \& Soares 2002).

Visando uma melhor caracterização da composição florística de trepadeiras nas florestas estacionais semidecíduas, foi realizado o levantamento dessas espécies em um fragmento localizado nos municípios de Araras e Rio Claro (SP) e analisada a variação desse componente florístico em comparação com outros levantamentos existentes para essas formações vegetais do Estado de São Paulo.

\section{Material e métodos}

Caracterização da área de estudo - A área de estudo compreende um fragmento de aproximadamente 230 hectares de floresta (Pagano \& Leitão Filho 1987), localizado na Fazenda São José, abrangendo partes dos municípios de Rio Claro e Araras (22 $21^{\prime} \mathrm{S}$ e $47^{\circ} 28^{\prime}-47^{\circ} 29^{\prime} \mathrm{W}, 630 \mathrm{~m}$ de altitude). Na região, o clima é do tipo Cwa segundo a classificação de Köppen (Nimer 1989), e conta com duas estações bem definidas: uma seca, de abril a setembro e outra chuvosa, de outubro a março. A precipitação média anual é da ordem de $1.360 \mathrm{~mm}$ e as temperaturas do mês mais frio variam entre $3 \mathrm{e}$ $18^{\circ} \mathrm{C}$ (Pagano \& Leitão Filho 1987).

$\mathrm{Na}$ área de estudo o terreno é relativamente plano e os solos são dos tipos Latossolo Vermelho-Amarelo e Latossolo Vermelho-Escuro, apresentando-se com boa fertilidade (Pagano \& Leitão Filho 1987).

A vegetação, que se caracteriza como uma floresta estacional semidecídua (segundo Rizzini 1963, floresta estacional mesófila semidecídua), possui dossel mais ou menos denso em função dos diferentes graus de perturbação. O componente arbóreo se apresenta em dois estratos floristicamente distintos (Pagano et al. 1987). O estrato superior, que se define entre 7 e $15 \mathrm{~m}$, além de emergentes que alcançam 20-25 m, é mais diversificado e tem como famílias mais importantes Leguminosae, Euphorbiaceae, Lauraceae, Anacardiaceae e Arecaceae (Pagano et al. 1987). O estrato inferior, com até $7 \mathrm{~m}$, conta com o predomínio de representantes das famílias Rutaceae, Meliaceae, Euphorbiaceae e Rubiaceae (Pagano et al. 1987). No campo, observa-se que, entre as formas subarbustivas e herbáceas, são freqüentes os representantes das famílias Rubiaceae, Acanthaceae e Poaceae. O componente das plantas epífitas e hemiepífitas pouco se destaca no conjunto da vegetação, enquanto que as trepadeiras salientam-se, principalmente nas bordas e nos locais de maiores clareiras.

Procedimentos - Para o levantamento florístico das trepadeiras foram realizadas coletas mensais durante o período de setembro de 2000 a abril de 2002 . Foram percorridas trilhas pré-existentes e bordas da floresta, coletando-se materiais de espécies em estádio reprodutivo. Os materiais coletados foram herborizados e, posteriormente, depositados no Herbário Rioclarense (HRCB). Concomitantemente, foram feitas as identificações utilizando-se bibliografia específica e comparações com materiais dos herbários HRCB, SP e UEC. Eventualmente, foram feitas consultas a especialistas. Os táxons foram listados segundo o sistema de Cronquist (1988), excetuando-se as Leguminosae que tradicionalmente têm sido tratadas como uma única família (Polhill \& Raven 1981). Para as abreviações dos autores das espécies utilizaram-se as recomendações de Brummitt \& Powell (1992).

Foram consideradas trepadeiras todas as plantas fanerógamas que necessitavam de um suporte para o seu desenvolvimento e que mantinham um contato permanente com o solo (Hegarty 1991), excluindo-se, portanto, as plantas hemiepífitas e epífitas. Considerando-se que as trepadeiras apresentam grande diversidade e plasticidade morfológica, buscou-se classificá-las em categorias que, geralmente, se relacionaram às posições que estas plantas ocupam na floresta e que auxiliasse na própria distinção entre as plantas trepadoras e as não trepadoras. Dessa forma, com base na estrutura caulinar, as espécies encontradas foram divididas nas categorias de trepadeiras herbáceas, quando apresentavam caules delgados e não lenhosos, ou trepadeiras lenhosas, quando apresentavam caules lenhosos 
freqüentemente de maior diâmetro. Usualmente, essas duas categorias de trepadeiras ocupam diferentes nichos. Trepadeiras herbáceas ("vines") geralmente desenvolvemse em áreas degradadas como bordas de floresta e clareiras e, em função de seus caules, normalmente apresentam crescimento limitado em extensão (geralmente menor que $5 \mathrm{~m}$ ) e encontram-se próximas do solo ou sobre arbustos e árvores de pequeno porte (Lima et al. 1997, Haber 2000). Trepadeiras lenhosas, ou lianas propriamente ditas (Gentry 1991), têm caules mais longos e são mais freqüentes sobre as copas das árvores (Morellato \& Leitão Filho 1998, Haber 2000).

Além de serem agrupadas nas duas categorias supra citadas, as espécies foram classificadas quanto às suas adaptações mecânicas (formas de escalar ou estruturas de fixação) segundo Kelly (1985) e Hegarty (1991), ligeiramente modificado: (1) trepadeiras volúveis - nessa categoria foram incluídas as plantas que se enrolavam em torno de um suporte por meio de ramos, caule e, mais raramente, pecíolos; (2) trepadeiras com gavinhas - nessa categoria foram agrupadas as plantas que se fixavam a um suporte por meio de estruturas modificadas em gavinhas de origem diversa (caulinar, foliar, etc.); e, (3) trepadeiras não preensoras ou passivas - nessa categoria foram tratadas as plantas que não possuíam estruturas específicas de fixação ou sensibilidade em órgãos, tais como ramos ou pecíolos que se enrolam sobre a planta suporte (forófito), e escalavam apoiando-se passivamente sobre um suporte. Na prática, essa é a classe que oferece maior grau de dificuldade para ser reconhecida, uma vez que muitas plantas podem apresentar-se com formas intermediárias entre ereta e escandente, o que se dá em função do tempo de desenvolvimento e das variações ambientais. Nesses casos, foram consideradas como trepadeiras não preensoras todas as plantas que apresentavam ramos estendidos e flexuosos, geralmente se fixando sobre outras por meio de ramificações laterais perpendiculares ao eixo principal, acúleos ou espinhos.

\section{Resultados}

Foram amostradas 148 espécies de trepadeiras, distribuídas em 33 famílias e 82 gêneros (tabela 1).

Foram identificadas quatro famílias pertencentes às monocotiledôneas (Alstroemeriaceae, Commelinaceae, Dioscoriaceae e Smilacaceae) das quais apenas Smilacaceae esteve representada por mais de uma espécie (três). As dicotiledôneas, portanto, abrangeram quase o total das espécies levantadas $(96 \%)$ e a ampla maioria das famílias encontradas (88\%).

Entre as famílias, Bignoniaceae destacou-se pelo elevado número de espécies (29 espécies) (tabela 1). As outras famílias mais representadas em número de espécies foram Asteraceae (19 espécies), Sapindaceae (12), Malpighiaceae (11) e Convolvulaceae (nove) que, em conjunto com Bignoniaceae, representaram 54\% das espécies encontradas neste levantamento. Por outro lado, $76 \%$ das famílias apresentaram menos de cinco espécies, e nove famílias (27\%) foram registradas com uma única espécie.

Bignoniaceae e Asteraceae, as duas famílias mais ricas em espécies, também apresentaram maior número de gêneros, 16 e oito, respectivamente (tabela 1). Entre os gêneros, os mais ricos em espécies foram Arrabidaea (com oito espécies), Ipomoea, Mikania e Serjania (com sete espécies cada). Esses gêneros pertencem às famílias que estão entre aquelas de maior riqueza específica: Bignoniaceae, Convolvulaceae, Asteraceae e Sapindaceae. Saliente-se o fato da maioria dos gêneros $(62 \%)$ ter sido representada por uma única espécie.

Quanto às diferentes categorias de trepadeiras, verificou-se que $63 \%$ das espécies eram lenhosas, enquanto as formas herbáceas representaram somente $37 \%$ das espécies (tabela 1).

Em relação aos mecanismos de escalar, constatouse o predomínio de espécies volúveis, perfazendo um total de $43 \%$ das espécies, seguido pelas formas dotadas de gavinhas (39\%) e, em menor número, pelas trepadeiras não preensoras, com 18\% das espécies (tabela 1).

\section{Discussão}

O número de espécies de trepadeiras encontrado nesse fragmento de floresta semidecídua evidencia a importante participação desse componente na diversidade vegetal dessa formação, se comparado com o total de espécies arbustivo-arbóreas (234 espécies) reconhecidas nessa mesma área (Pagano \& Leitão Filho 1987, Pagano et al. 1995).

Outros levantamentos voltados exclusivamente para as trepadeiras, realizados em florestas estacionais semidecíduas no Estado de São Paulo (tabela 2), apontaram a expressiva riqueza desse componente (105 a 136 espécies). Saliente-se o fato desses estudos terem abrangido fragmentos de florestas de proporções consideravelmente limitadas (112 a 250 hectares). Sendo assim, pode-se considerar que a elevada riqueza de trepadeiras é um importante atributo desses fragmentos de florestas estacionais semidecíduas.

A grande quantidade de espécies de trepadeiras encontrada nesses estudos foi atribuída, em parte, à heterogeneidade de habitats (Hora \& Soares 2002), à situação de fragmento em que esses remanescentes 
Tabela 1. Trepadeiras da floresta estacional semidecídua, Fazenda São José, Rio Claro / Araras, SP; suas formas de fixação (Gav. = com gavinhas; $\mathrm{NP}=$ não preensora; Vol. = volúvel), hábito $(\mathrm{H}=$ herbáceo; $\mathrm{L}=$ lenhoso $)$ e material testemunho do HRCB, segundo número do coletor (A = M.A. Assis; P = D.G. Picchi e U = R.G. Udulutsch).

Table 1. Climber species in a seasonal semi-deciduous forest, São José farm, Rio Claro / Araras, São Paulo state, Brazil; patterns of climbing (Gav. = with tendrils; $\mathrm{NP}=$ scandent; Vol. = twines $)$, habits $(\mathrm{H}=$ herbaceous; $\mathrm{L}=$ woody lianas $)$ and voucher's number of HRCB according collectors ( $\mathrm{A}=$ M.A. Assis; $\mathrm{P}=$ D.G. Picchi and $\mathrm{U}=$ R.G. Udulutsch).

\begin{tabular}{|c|c|c|c|}
\hline Família/Espécie & Estrutura de fixação & Hábito & Material (HRCB) \\
\hline \multicolumn{4}{|l|}{ ALSTROEMERIACEAE } \\
\hline Alstroemeria nemorosa Gardner & Vol. & $\mathrm{H}$ & $\mathrm{U} / 247$ \\
\hline \multicolumn{4}{|l|}{ AMARANTHACEAE } \\
\hline Chamissoa macrocarpa Kunth & $\mathrm{NP}$ & $\mathrm{H}$ & $\mathrm{U} / 301$ \\
\hline \multicolumn{4}{|l|}{ APOCYNACEAE } \\
\hline Condylocarpon isthimicum (Vell.) A. DC. & Vol. & $\mathrm{L}$ & $\mathrm{U} / 171$ \\
\hline Forsteronia leptocarpa (Hook. \& Arn.) A. DC. & Vol. & $\mathrm{L}$ & $\mathrm{U} / 285$ \\
\hline Forsteronia pilosa (Vell.) Müll. Arg. & Vol. & $\mathrm{L}$ & $\mathrm{U} / 198$ \\
\hline Forteronia pubescens A. DC. & Vol. & $\mathrm{L}$ & $\mathrm{U} / 214$ \\
\hline Forsteronia refracta Müll. Arg. & Vol. & $\mathrm{L}$ & $\mathrm{U} / 110$ \\
\hline Peltastes sp. & Vol. & $\mathrm{L}$ & $\mathrm{U} / 153$ \\
\hline Prestonia coalita (Vell.) Woodson & Vol. & $\mathrm{H}$ & $\mathrm{U} / 163$ \\
\hline \multicolumn{4}{|l|}{ ARISTOLOCHIACEAE } \\
\hline Aristolochia arcuata W. Mast. & Vol. & $\mathrm{H}$ & $\mathrm{U} / 155$ \\
\hline Aristolochia galeata Mart. \& Zucc. & Vol. & $\mathrm{L}$ & $\mathrm{U} / 349$ \\
\hline Aristolochia melastoma Manso ex Duch. & Vol. & $\mathrm{H}$ & $\mathrm{U} / 184$ \\
\hline \multicolumn{4}{|l|}{ ASCLEPIADACEAE } \\
\hline Gonioanthela hilariana (E. Fourn.) Malme & Vol. & $\mathrm{H}$ & $\mathrm{U} / 99$ \\
\hline Gonolobus selloanus (E. Fourn.) Bacigalupo & Vol. & $\mathrm{H}$ & $\mathrm{U} / 194$ \\
\hline Orthosia urceolata E. Fourn. & Vol. & $\mathrm{H}$ & $\mathrm{A} / 1357$ \\
\hline \multicolumn{4}{|l|}{ ASTERACEAE } \\
\hline Baccharis flexuosa Baker & NP & $\mathrm{L}$ & $\mathrm{U} / 207$ \\
\hline Bidens squarrosa Kunth & Vol. & $\mathrm{H}$ & $\mathrm{U} / 126$ \\
\hline Calea pinnatifida Less. & NP & $\mathrm{H}$ & $\mathrm{U} / 424$ \\
\hline Eupatorium maximilianii Schrad. \& DC. & NP & $\mathrm{L}$ & $\mathrm{U} / 295$ \\
\hline Eupatorium megaphyllum Baker & $\mathrm{NP}$ & $\mathrm{L}$ & $\mathrm{U} / 380$ \\
\hline Eupatorium vauthierianum DC. & NP & $\mathrm{H}$ & $\mathrm{U} / 302$ \\
\hline Eupatorium vitalbae DC. & Vol. & $\mathrm{H}$ & $\mathrm{A} / 1348$ \\
\hline Mikania glomerata Spreng. & Vol. & $\mathrm{L}$ & $\mathrm{U} / 405$ \\
\hline Mikania micrantha Kunth & Vol. & $\mathrm{H}$ & $\mathrm{U} / 297$ \\
\hline Mikania pyramidata Donn. \& Sm. & Vol. & $\mathrm{L}$ & $\mathrm{U} / 401$ \\
\hline Mikania ramosissima Gardner & Vol. & $\mathrm{L}$ & $\mathrm{U} / 389$ \\
\hline Mikania triangularis Baker & Vol. & $\mathrm{L}$ & A / 1377 \\
\hline Mikania sp. 1 & Vol. & $\mathrm{H}$ & $\mathrm{U} / 348$ \\
\hline Mikania sp. 2 & Vol. & $\mathrm{H}$ & $\mathrm{U} / 477$ \\
\hline Mutisia coccinea A. St.-Hil. & Gav. & $\mathrm{L}$ & $\mathrm{U} / 393$ \\
\hline Trixis divaricata (Kunth) Spreng. & $\mathrm{NP}$ & $\mathrm{H}$ & $\mathrm{U} / 45$ \\
\hline Vernonia crassa (Vell.) Ekman \& Malme & $\mathrm{NP}$ & $\mathrm{L}$ & $\mathrm{U} / 370$ \\
\hline Vernonia diffusa Less. & $\mathrm{NP}$ & $\mathrm{L}$ & $\mathrm{A} / 1370$ \\
\hline Vernonia scorpioides (Lam.) Pers. & $\mathrm{NP}$ & $\mathrm{L}$ & $\mathrm{U} / 87$ \\
\hline \multicolumn{4}{|l|}{ BIGNONIACEAE } \\
\hline Adenocalymma bracteatum (Cham.) A. DC. & Gav. & $\mathrm{L}$ & $\mathrm{U} / 326$ \\
\hline Adenocalymma marginatum (Cham.) A. DC. & Gav. & $\mathrm{L}$ & $\mathrm{U} / 82$ \\
\hline Adenocalymma paulistarum Bureau \& K. Schum. & Gav. & $\mathrm{L}$ & $\mathrm{U} / 395$ \\
\hline Amphilophium paniculatum (L.) Kunth & Gav. & $\mathrm{L}$ & $\mathrm{U} / 131$ \\
\hline
\end{tabular}




\begin{tabular}{|c|c|c|c|}
\hline Família/Espécie & Estrutura de fixação & Hábito & Material (HRCB) \\
\hline \multicolumn{4}{|l|}{ BIGNONIACEAE } \\
\hline Anemopaegma chamberlaynii (Sims) Bureau \& K. Schum. & Gav. & $\mathrm{L}$ & $\mathrm{U} / 120$ \\
\hline Arrabidaea chica (Bonpl.) Verl. & Gav. & $\mathrm{L}$ & $\mathrm{U} / 154$ \\
\hline Arrabidaea formosa (Bureau) Sandwith & Gav. & $\mathrm{L}$ & $\mathrm{U} / 329$ \\
\hline Arrabidaea pubescens (L.) A.H. Gentry & Gav. & $\mathrm{L}$ & $\mathrm{U} / 352$ \\
\hline Arrabidaea pulchella (Cham.) Bureau & Gav. & $\mathrm{L}$ & $\mathrm{U} / 32$ \\
\hline Arrabidaea pulchra (Cham.) Sandwith & Gav. & $\mathrm{L}$ & $\mathrm{U} / 284$ \\
\hline Arrabidaea samydoides (Cham.) Sandwith & Gav. & $\mathrm{L}$ & $\mathrm{U} / 234$ \\
\hline Arrabidaea selloi (Spreng.) Sandwith & Gav. & $\mathrm{L}$ & $\mathrm{U} / 84$ \\
\hline Arrabidaea triplinervia (Mart. ex A. DC.) Baill. ex Bureau & Gav. & $\mathrm{L}$ & $\mathrm{U} / 216$ \\
\hline Clytostoma campanulatum (Cham.) Bureau \& K. Schum. & Gav. & $\mathrm{L}$ & $\mathrm{U} / 121$ \\
\hline Clytostoma sciuripabulum Bureau \& K. Schum. & Gav. & $\mathrm{L}$ & $\mathrm{U} / 33$ \\
\hline Cuspidaria convoluta (Vell.) A.H. Gentry & Gav. & $\mathrm{L}$ & A/1499 \\
\hline Cuspidaria floribunda (A. DC.) A.H. Gentry & Gav. & $\mathrm{L}$ & $\mathrm{U} / 209$ \\
\hline Fridericia speciosa Mart. & Gav. & $\mathrm{L}$ & $\mathrm{U} / 350$ \\
\hline Lundia obliqua Sond. & Gav. & $\mathrm{L}$ & $\mathrm{U} / 221$ \\
\hline Macfadyena mollis (Sond.) Seem. & Gav. & $\mathrm{L}$ & $\mathrm{U} / 41$ \\
\hline Macfadyena unguis-cati (L.) A.H. Gentry & Gav. & $\mathrm{L}$ & $\mathrm{A} / 1365$ \\
\hline Mansoa difficilis (Cham.) Bureau \& K. Schum. & Gav. & $\mathrm{L}$ & $\mathrm{U} / 83$ \\
\hline Melloa quadrivalvis (Jacq.) A.H. Gentry & Gav. & $\mathrm{L}$ & A/ 1495 \\
\hline Pithecoctenium crucigerum (L.) A.H. Gentry & Gav. & $\mathrm{L}$ & $\mathrm{U} / 396$ \\
\hline Pleonotoma tetraquetrum (Cham.) Bureau & Gav. & $\mathrm{L}$ & A/ 1657 \\
\hline Pyrostegia venusta (Ker-Gawl.) Miers & Gav. & $\mathrm{L}$ & $\mathrm{U} / 357$ \\
\hline Stizophyllum perforatum (Cham.) Miers & Gav. & $\mathrm{L}$ & $\mathrm{U} / 220$ \\
\hline Tynanthus elegans (Cham.) Miers & Gav. & $\mathrm{L}$ & $\mathrm{U} / 101$ \\
\hline Tynanthus fasciculatus (Vell.) Miers & Gav. & $\mathrm{L}$ & $\mathrm{U} / 57$ \\
\hline \multicolumn{4}{|l|}{ BORAGINACEAE } \\
\hline Tournefortia elegans Cham. & $\mathrm{NP}$ & $\mathrm{L}$ & $\mathrm{U} / 48$ \\
\hline Tournefortia paniculata Cham. & NP & $\mathrm{L}$ & $\mathrm{U} / 94$ \\
\hline Tournefortia sp. & NP & $\mathrm{L}$ & $\mathrm{P} / 96$ \\
\hline \multicolumn{4}{|l|}{ CACTACEAE } \\
\hline Pereskia aculeata Mill. & $\mathrm{NP}$ & $\mathrm{L}$ & $\mathrm{U} / 222$ \\
\hline \multicolumn{4}{|l|}{ COMMELINACEAE } \\
\hline Dichorisandra hexandra (Aubl.) Standl. & $\mathrm{NP}$ & $\mathrm{H}$ & $\mathrm{P} / 77$ \\
\hline \multicolumn{4}{|l|}{ CONVOLVULACEAE } \\
\hline Ipomoea aff. brasiliana (Choisy) Meisn. & Vol. & $\mathrm{H}$ & $\mathrm{U} / 217$ \\
\hline Ipomoea heredifolia $\mathrm{L}$. & Vol. & $\mathrm{H}$ & $\mathrm{P} / 114$ \\
\hline Ipomoea nil (L.) Roth & Vol. & $\mathrm{H}$ & $\mathrm{U} / 185$ \\
\hline Ipomoea quamoclit $\mathrm{L}$. & Vol. & $\mathrm{H}$ & $\mathrm{U} / 193$ \\
\hline Ipomoea saopaulista O’Donell & Vol. & $\mathrm{H}$ & $\mathrm{U} / 299$ \\
\hline Ipomoea syringaefolia Meisn. & Vol. & $\mathrm{H}$ & $\mathrm{U} / 224$ \\
\hline Iротоеа sp. & Vol. & $\mathrm{H}$ & $\mathrm{P} / 102$ \\
\hline Jacquemontia ciliata Sandwith & Vol. & $\mathrm{H}$ & $\mathrm{U} / 168$ \\
\hline Merremia macrocalyx (Ruiz \& Pav.) O’Donell & Vol. & $\mathrm{H}$ & $\mathrm{U} / 303$ \\
\hline \multicolumn{4}{|l|}{ CUCURBITACEAE } \\
\hline Psiguria ternata (Roem.) C. Jeffrey & Gav. & $\mathrm{H}$ & $\mathrm{P} / 27$ \\
\hline Psiguria warmingiana (Cogn.) C. Jeffrey & Gav. & $\mathrm{H}$ & U/191 \\
\hline Cucumis sp. & Gav. & $\mathrm{H}$ & $\mathrm{P} / 113$ \\
\hline Momordica charantia L. & Gav. & $\mathrm{H}$ & $\mathrm{U} / 132$ \\
\hline Wilbrandia longibracteata Cogn. & Gav. & $\mathrm{H}$ & $\mathrm{U} / 237$ \\
\hline \multicolumn{4}{|l|}{ DILLENIACEAE } \\
\hline Davilla rugosa Poir. & Vol. & $\mathrm{L}$ & $\mathrm{U} / 49$ \\
\hline
\end{tabular}


continuação

\begin{tabular}{|c|c|c|c|}
\hline Família/Espécie & Estrutura de fixação & Hábito & Material (HRCB) \\
\hline \multicolumn{4}{|l|}{ DIOSCOREACEAE } \\
\hline Dioscorea dodecaneura Vell. & Vol. & $\mathrm{H}$ & $\mathrm{U} / 349$ \\
\hline \multicolumn{4}{|l|}{ EUPHORBIACEAE } \\
\hline Dalechampia scandens $\mathrm{L}$. & Vol. & $\mathrm{H}$ & $\mathrm{U} / 192$ \\
\hline Dalechampia stipulacea Müll. Arg. & Vol. & $\mathrm{H}$ & $\mathrm{U} / 96$ \\
\hline Dalechampia triphylla Lam. & Vol. & $\mathrm{H}$ & $\mathrm{U} / 341$ \\
\hline Tragia sellowiana (Baill.) Müll. Arg. & Vol. & $\mathrm{H}$ & $\mathrm{U} / 116$ \\
\hline \multicolumn{4}{|l|}{ HIPPOCRATEACEAE } \\
\hline Anthodon decussata Ruiz \& Pav. & Vol. & $\mathrm{L}$ & $\mathrm{U} / 111$ \\
\hline Hippocratea volubilis L. & Vol. & $\mathrm{L}$ & $\mathrm{U} / 354$ \\
\hline \multicolumn{4}{|l|}{ LEGUMINOSAE } \\
\hline Acacia paniculata Willd. & Vol. & $\mathrm{L}$ & $\mathrm{U} / 106$ \\
\hline \multirow[t]{2}{*}{ Centrosema sagittatum (Humb. \& Bonpl. ex Willd.) } & Vol. & $\mathrm{H}$ & $\mathrm{U} / 277$ \\
\hline & \multicolumn{2}{|c|}{ Brandegee ex L. Riley } & \\
\hline Machaerium oblongifolium Vogel & NP & $\mathrm{L}$ & $\mathrm{U} / 78$ \\
\hline Phaseolus sp. 1 & Vol. & $\mathrm{H}$ & $\mathrm{U} / 215$ \\
\hline Phaseolus sp. 2 & Vol. & $\mathrm{H}$ & $\mathrm{P} / 99$ \\
\hline \multicolumn{4}{|l|}{ MALPIGHIACEAE } \\
\hline Banisteriopsis adenopoda (A. Juss.) B. Gates & Vol. & $\mathrm{L}$ & $\mathrm{U} / 291$ \\
\hline Banisteriopsis lutea (Griseb.) Cuatrec. & Vol. & $\mathrm{L}$ & $\mathrm{A} / 1342$ \\
\hline Banisteriopsis oxyclada (A. Juss.) B. Gates & Vol. & $\mathrm{L}$ & $\mathrm{U} / 288$ \\
\hline Dicella bracteosa (A. Juss.) Griseb. & Vol. & $\mathrm{L}$ & $\mathrm{U} / 79$ \\
\hline Heteropterys birsonimifolia A. Juss. & Vol. & $\mathrm{L}$ & A/ 1494 \\
\hline Heteropterys intermedia (A. Juss.) Griseb. & Vol. & $\mathrm{L}$ & $\mathrm{U} / 279$ \\
\hline Heteropterys sp. & Vol. & $\mathrm{L}$ & $\mathrm{P} / 97$ \\
\hline Mascagnia cordifolia (A. Juss.) Griseb. & Vol. & $\mathrm{L}$ & $\mathrm{U} / 475$ \\
\hline Mascagnia cf. lasiandra Nied. & Vol. & $\mathrm{L}$ & $\mathrm{U} / 402$ \\
\hline Stigmaphyllon lalandianum A. Juss. & Vol. & $\mathrm{L}$ & $\mathrm{U} / 384$ \\
\hline Tetrapterys phlomoides (Spreng.) Nied. & Vol. & $\mathrm{L}$ & $\mathrm{U} / 188$ \\
\hline \multicolumn{4}{|l|}{ MENDONCIACEAE } \\
\hline Mendoncia puberula Mart. & Vol. & $\mathrm{H}$ & $\mathrm{U} / 320$ \\
\hline Mendoncia velloziana Mart. & Vol. & $\mathrm{H}$ & $\mathrm{P} / 20$ \\
\hline \multicolumn{4}{|l|}{ MENISPERMACEAE } \\
\hline Cissampelos andromorpha DC. & Vol. & $\mathrm{H}$ & $\mathrm{A} / 1503$ \\
\hline Cissampelos glaberrima A. St.-Hil. & Vol. & $\mathrm{H}$ & $\mathrm{U} / 61$ \\
\hline Cissampelos pareira $\mathrm{L}$. & Vol. & $\mathrm{H}$ & $\mathrm{U} / 105$ \\
\hline Odontocarya acuparata Miers & Vol. & $\mathrm{H}$ & $\mathrm{U} / 109$ \\
\hline \multicolumn{4}{|l|}{ PASSIFLORACEAE } \\
\hline Passiflora capsularis L. & Gav. & $\mathrm{H}$ & $\mathrm{U} / 426$ \\
\hline Passiflora amethystina J.C. Mikan & Gav. & $\mathrm{H}$ & $\mathrm{P} / 103$ \\
\hline Passiflora sp. & Gav. & $\mathrm{H}$ & $\mathrm{P} / 89$ \\
\hline \multicolumn{4}{|l|}{ POLYGALACEAE } \\
\hline Bredemeyera floribunda Willd. & NP & $\mathrm{L}$ & $\mathrm{U} / 292$ \\
\hline Diclidanthera laurifolia Mart. & Vol. & $\mathrm{L}$ & A/ 1493 \\
\hline \multicolumn{4}{|l|}{ RANUNCULACEAE } \\
\hline Clematis dioica $\mathrm{L}$. & Vol. & $\mathrm{L}$ & $\mathrm{U} / 274$ \\
\hline \multicolumn{4}{|l|}{ RHAMNACEAE } \\
\hline Gouania latifolia Reissek & Gav. & $\mathrm{L}$ & $\mathrm{U} / 236$ \\
\hline Gouania virgata Reissek & Gav. & $\mathrm{L}$ & $\mathrm{U} / 363$ \\
\hline \multicolumn{4}{|l|}{ ROSACEAE } \\
\hline Rubus urticifolius Poir. & NP & $\mathrm{L}$ & $\mathrm{U} / 358$ \\
\hline
\end{tabular}


continuação

\begin{tabular}{|c|c|c|c|}
\hline Família/Espécie & Estrutura de fixação & Hábito & Material (HRCB) \\
\hline \multicolumn{4}{|l|}{ RUBIACEAE } \\
\hline Chiococca brachiata Ruiz \& Pav. & $\mathrm{NP}$ & $\mathrm{L}$ & $\mathrm{U} / 166$ \\
\hline Manettia cordifolia Mart. & Vol. & $\mathrm{H}$ & $\mathrm{P} / 32$ \\
\hline \multicolumn{4}{|l|}{ SAPINDACEAE } \\
\hline Cardiospermum grandiflorum $\mathrm{Sw}$. & Gav. & $\mathrm{L}$ & $\mathrm{U} / 364$ \\
\hline Paullinia elegans Cambess. & Gav. & $\mathrm{L}$ & $\mathrm{U} / 147$ \\
\hline Paullinia spicata Benth. & Gav. & $\mathrm{L}$ & $\mathrm{U} / 153$ \\
\hline Serjania caracasana (Jacq.) Willd. & Gav. & $\mathrm{L}$ & $\mathrm{U} / 374$ \\
\hline Serjania fuscifolia Radlk. & Gav. & $\mathrm{L}$ & $\mathrm{U} / 385$ \\
\hline Serjania glabrata Kunth & Gav. & $\mathrm{L}$ & $\mathrm{U} / 152$ \\
\hline Serjania laruotteana Cambess. & Gav. & $\mathrm{L}$ & $\mathrm{U} / 379$ \\
\hline Serjania lethalis A. St.-Hil. & Gav. & $\mathrm{L}$ & $\mathrm{A} / 1501$ \\
\hline Serjania meridionalis Cambess. & Gav. & $\mathrm{L}$ & $\mathrm{P} / 108$ \\
\hline Serjania reticulata Cambess. & Gav. & $\mathrm{L}$ & $\mathrm{U} / 289$ \\
\hline Urvillea laevis Radlk. & Gav. & $\mathrm{L}$ & $\mathrm{U} / 317$ \\
\hline Urvillea ulmacea Kunth & Gav. & $\mathrm{L}$ & $\mathrm{A} / 1360$ \\
\hline \multicolumn{4}{|l|}{ SMILACACEAE } \\
\hline Smilax elastica Griseb. & Gav. & $\mathrm{H}$ & $\mathrm{U} / 434$ \\
\hline Smilax fluminensis Steud. & Gav. & $\mathrm{H}$ & $\mathrm{U} / 142$ \\
\hline Smilax rufescens Griseb. & Gav. & $\mathrm{H}$ & $\mathrm{A} / 1500$ \\
\hline \multicolumn{4}{|l|}{ SOLANACEAE } \\
\hline Solanum alternatopinnatum Steud. & NP & $\mathrm{L}$ & $\mathrm{U} / 151$ \\
\hline Solanum swartzianum Roem. \& Schult. & $\mathrm{NP}$ & $\mathrm{L}$ & $\mathrm{U} / 283$ \\
\hline \multicolumn{4}{|l|}{ STERCULIACEAE } \\
\hline Byttneria australis A. St.-Hil. & NP & $\mathrm{L}$ & $\mathrm{U} / 119$ \\
\hline Byttneria catalpifolia Jacq. & $\mathrm{NP}$ & $\mathrm{L}$ & $\mathrm{P} / 29$ \\
\hline \multicolumn{4}{|l|}{ ULMACEAE } \\
\hline Celtis iguanae (Jacq.) Sarg. & $\mathrm{NP}$ & $\mathrm{L}$ & $\mathrm{U} / 251$ \\
\hline \multicolumn{4}{|l|}{ VERBENACEAE } \\
\hline Aloysia virgata (Ruiz \& Pav.) A. Juss. & $\mathrm{NP}$ & $\mathrm{L}$ & A/1341 \\
\hline Petrea subserrata Cham. & NP & $\mathrm{L}$ & A/ 1351 \\
\hline \multicolumn{4}{|l|}{ VITACEAE } \\
\hline Cissus serroniana (Glaz.) J.A. Lombardi & Gav. & $\mathrm{H}$ & $\mathrm{P} / 17$ \\
\hline Cissus sulcicaulis (Baker) Planch. & Gav. & $\mathrm{H}$ & $\mathrm{P} / 80$ \\
\hline Cissus verticillata (L.) Nicolson \& C.E. Jarvis & Gav. & $\mathrm{H}$ & $\mathrm{U} / 88$ \\
\hline
\end{tabular}

florestais se encontram (Morellato \& Leitão Filho 1998) e às perturbações que podem ter causas naturais ou antrópicas (Rezende 1997, Hora \& Soares 2002). Tanto o processo de fragmentação da floresta como os eventos de perturbação proporcionam um aumento de áreas com maior incidência luminosa, como clareiras e bordas, que favorecem o desenvolvimento dessa forma de vida (Morellato \& Leitão Filho 1996, 1998). Na área do presente estudo as condições acima citadas, somadas à fertilidade do solo, foram relatadas como determinantes da diversidade arbórea (Pagano \& Leitão Filho 1987), e podem responder, em parte, pelo elevado número de espécies de trepadeiras amostrado.
Em termos taxonômicos, verificou-se que as cinco famílias mais ricas contribuíram com 54\% das espécies inventariadas. Esse fato corrobora grande parte dos estudos de mesma natureza realizados em florestas tropicais, que relatam algumas poucas famílias representadas por grande número de espécies (Gentry 1988, Lima et al. 1997, Morellato \& Leitão Filho 1998, Ribeiro et al. 1999). Segundo Gentry (1991), embora muitas famílias possuam espécies com essa forma de vida (pelo menos 97 famílias de plantas com sementes no Novo Mundo), a grande maioria das espécies de trepadeiras concentra-se em um número relativamente pequeno de famílias, a ponto de 26 famílias deterem 
Tabela 2. Número de espécies de trepadeiras em fragmentos de floresta estacional semidecídua do Estado de São Paulo e de espécies em comum (compartilhadas) com a área do presente estudo.

Table 2. Number of climber species in seasonal semi-deciduous forests fragments of São Paulo state and number of species sharing the studied area.

\begin{tabular}{lcccl}
\hline Local de Estudo & Área (ha) & N. de espécies & Espécies em comum & Referência \\
\hline Campinas, SP & 250 & 136 & 61 & Morellato \& Leitão Filho 1998 \\
Rio Claro / Araras, SP & 230 & 148 & - & Presente estudo \\
São Carlos, SP & 112 & 109 & 38 & Hora 1999 \\
São José do Rio Preto, SP & 168,6 & 105 & 35 & Rezende 1997 \\
\hline
\end{tabular}

$85 \%$ do total de espécies de trepadeiras no Novo Mundo.

A constatação de que a família Bignoniaceae foi a mais rica, englobando $20 \%$ do total de espécies amostradas, reafirma outros relatos de que se trata da família mais importante em número de espécies de trepadeiras nesse tipo de vegetação. Segundo Gentry (1991), considerando uma série de amostragens incluindo apenas lianas (DAP maior ou igual a 2,5 cm), Bignoniaceae figura como a família mais rica na maioria dos estudos realizados em florestas neotropicais de baixas altitudes ("lowland forests"), nas quais se enquadram as florestas estacionais semidecíduas, ocorrendo, dessa maneira, sob diferentes condições climáticas e solos variados. Nos fragmentos de floresta estacional semidecídua do Estado de São Paulo, o maior número de espécies de trepadeiras para as Bignoniaceae tem sido apontado invariavelmente, mesmo nos estudos que consideraram tanto as trepadeiras lenhosas (lianas) como as herbáceas (Rezende 1997, Morellato \& Leitão Filho 1998, Hora \& Soares 2002).

Considerando-se as demais famílias mais ricas na área de estudo, Sapindaceae, Malpighiaceae e Apocynaceae, estiveram representadas principalmente por espécies lenhosas. As duas primeiras, assim como Bignoniaceae, também se destacam em termos de riqueza em lianas nas florestas tropicais de baixas altitudes, embora com menor importância (Gentry 1991). Entre aquelas famílias que estiveram representadas principalmente por trepadeiras herbáceas, ressalta-se a família Asteraceae, que tem sido relacionada, na maior parte das vezes, na segunda ou na terceira posições de riqueza em florestas estacionais semidecíduas do Estado de São Paulo (Rezende 1997, Morellato \& Leitão Filho 1998, Hora 1999). No entanto, segundo o estudo de Lima et al. (1997), Asteraceae apresenta diversidade ainda maior em floresta atlântica, corroborando o relato de
Gentry (1991) de que essa família torna-se preponderante em florestas tropicais montanas.

Em relação à distribuição dos gêneros, o fato de Bignoniaceae ser a família com o terceiro maior número de gêneros em trepadeiras no Novo Mundo (53 gêneros) e, sobretudo por ter o Brasil como um de seus centros de diversidade (Gentry 1980, 1991), seria um dos fatores determinantes para os resultados obtidos no presente estudo, em que a família agrupou $20 \%$ do total de gêneros amostrados. A maior diversidade de gêneros na família Bignoniaceae concorda com os resultados de outros estudos realizados em florestas estacionais semidecíduas (Morellato \& Leitão Filho 1998, Hora \& Soares 2002) e, portanto, essa parece ser uma das características na composição florística de trepadeiras dessa formação. De forma semelhante, os gêneros que foram amostrados com maior número de espécies no presente estudo, como Arrabidaea, Ipomoea, Mikania e Serjania, também figuram entre os mais ricos em outros levantamentos florísticos de trepadeiras realizados nesse tipo de floresta (e.g. Morellato \& Leitão Filho 1998).

De outra maneira, quando se comparam as espécies encontradas no presente estudo com os demais levantamentos de trepadeiras realizados em fragmentos de florestas estacionais semidecíduas no Estado de São Paulo (tabela 2), verifica-se que o número de espécies compartilhadas é relativamente baixo, variando de 35 a 61 espécies. Fatores como clima, altitude, solo e contexto geográfico, têm sido relatados como importantes para explicar diferenças de composição para as trepadeiras (Gentry 1988), mas pouco se conhece a respeito das variações florísticas do componente das trepadeiras nessas florestas do interior do Estado de São Paulo.

A verificação de que as trepadeiras lenhosas (lianas) foram preponderantes na área, enquanto as formas herbáceas representaram apenas $37 \%$ das espécies, está 
de acordo com outros resultados obtidos em florestas estacionais semidecíduas, como nos estudos de Bernacci \& Leitão Filho (1996) e Morellato \& Leitão Filho (1998), nos quais as lianas compuseram a categoria mais diversificada, representando cerca de $2 / 3$ das espécies de trepadeiras. No entanto, deve-se ressaltar que esses resultados se opõem à estimativa de Gentry (1991), segundo a qual as trepadeiras herbáceas teriam a mesma representatividade das trepadeiras lenhosas em florestas tropicais de baixa altitude, contribuindo, também, com cerca de $10 \%$ da riqueza dessa flora. De fato, como estimado por Gentry (1991), valores semelhantes entre as duas categorias de trepadeiras foram relatados para florestas equatorianas (Gentry \& Dodson 1987), na flora de Barro Colorado (Croat 1978) e na flora da Costa Rica (Janzen \& Liesner 1980). Em face à escassez de levantamentos incluindo as trepadeiras em florestas estacionais semidecíduas, pouco se pode concluir em relação a essas divergências de representatividade entre as categorias de trepadeiras herbáceas e lenhosas.

Apesar das diferentes formas de escalar das trepadeiras terem importância reconhecida, tanto do ponto de vista taxonômico quanto ecológico, raros são os estudos que buscaram classificar e quantificar esses mecanismos de ascensão nas espécies das formações vegetais brasileiras. No presente estudo, as formas não preensoras estiveram representadas em algumas famílias que, muitas vezes, não possuem órgãos específicos para escalar, como é o caso de Solanaceae, Cactaceae (ambas dotadas de espinhos) e Amaranthaceae (apresentando caules flexuosos com ramificações divaricadas). O fato das formas não preensoras comporem o menor contingente das espécies encontradas neste estudo (18\%), pode se relacionar, em parte, com o menor grau de especialização dos mecanismos de ascensão dessa categoria de trepadeiras, conforme proposto por Gentry (1991). Contudo, para que o baixo percentual das trepadeiras não preensoras e a supremacia das formas volúveis e com gavinhas sejam apontados como uma característica dessa formação, seria prudente analisar um maior número de estudos em fragmentos de florestas estacionais semidecíduas.

A evidência de que as trepadeiras podem representar grande parte da riqueza de espécies nessas florestas reforça a necessidade de novos estudos voltados para esse componente. Aparentemente, as trepadeiras apresentam semelhança florística nos fragmentos de florestas estacionais semidecíduas, no que diz respeito às famílias e gêneros mais ricos em espécies. Porém, há de se ressaltar que a composição, em nível específico, mostra-se bastante distinta e pouco se pode afirmar a respeito dessas variações florísticas sem que haja mais estudos.

Agradecimentos - Ao CNPq pela concessão de bolsa PIBIC ao primeiro e ao terceiro autores. Aos especialistas pelo auxílio nas identificações: Dr. João Semir (Asteraceae), Dra. Maria Cândida H. Mamede (Malpighiaceae) e Dra. Rosângela SimãoBianchini (Convolvulaceae). À Dra. L. Patrícia C. Morellato e revisores anônimos, pelas sugestões dadas ao manuscrito.

\section{Referências bibliográficas}

BERNACCI, L.C. \& LEITÃO FILHO, H.F. 1996. Flora fanerogâmica da fazenda São Vicente, Campinas, SP. Revista Brasileira de Botânica 19:149-164.

BRUMMITT, R.K. \& POWELL, C.E. 1992. Authors of plant names. Royal Botanic Gardens, Kew.

CLARK, D.B. \& CLARK, D.A. 1990. Distribution and effects on tree growth of lianas and woody hemiepiphytes in a Costa Rican tropical wet forest. Journal of Tropical Ecology 6:321-331.

CROAT, T.B. 1978. Flora of Barro Colorado Island. Stanford University Press, Stanford.

CRONQUIST, A. 1988. An integrated system of classification of flowering plants. Columbia University Press, New York.

ENGEL, V.L., FONSECA, R.C.B. \& OLIVEIRA, R.E. 1998. Ecologia de lianas e o manejo de fragmentos florestais. Série Técnica IPEF 12:43-64.

GENTRY, A.H. 1978. Diversidade e regeneração da capoeira do INPA, com referência especial às Bignoniaceae. Acta Amazonica 8:67-70.

GENTRY, A.H. 1980. Bignoniaceae. Part I (Crescentiae and Tourrettieae). Flora Neotropica, New York, v.25, p.1-130.

GENTRY, A.H. 1988. Changes in plant community diversity and floristic composition on environmental and geographical gradients. Annals of the Missouri Botanical Garden 75:1-34.

GENTRY, A.H. 1991. The distribution and evolution of climbing plants. In The Biology of vines (F.E. Putz \& H.A. Mooney, eds.). Cambridge University Press, Cambridge, p.3-49.

GENTRY, A.H. \& DODSON, C. 1987. Contribution of nontrees to species richness of a tropical rain forest. Biotropica 19:149-156.

HABER, W.A. 2000. Plants and vegetation. In Monteverde ecology and conservation of a tropical cloud forest (N.M. Nadkarni \& N.T. Wheelwright, eds.). Oxford University Press, New York, p.39-94.

HEGARTY, E.E. 1991. Vine-host interactions. In The Biology of vines (F.E. Putz \& H.A. Mooney, eds.). Cambridge University Press, Cambridge, p.357-375. 
HORA, R.C. 1999. Composição florística e aspectos da estrutura da comunidade de lianas em uma mata mesófila semidecídua na Fazenda Canchim, São Carlos - SP. Dissertação de mestrado, Universidade Federal de São Carlos, São Carlos.

HORA, R.C. \& SOARES, J.J. 2002. Estrutura fitossociológica da comunidade de lianas em uma floresta estacional semidecidual na Fazenda Canchim, São Carlos, SP. Revista Brasileira de Botânica 25:323-329.

JANZEN, D.H. \& LIESNER, R. 1980. Annoted checklist of lowland Guanacaste provincial Costa Rica, exclusive of grasses and nonvascular cryptogams. Brenesia 18:15-90.

KELLY, D.L. 1985. Epiphytes and climbers of a Jamaican rain forest: vertical distribution, life forms and life histories. Journal of Biogeography 12:223-241.

LIMA, H.C., LIMA, M.P.M., VAZ, A.M.S.F. \& PESSOA, S.V.A. 1997. Trepadeiras da Reserva Ecológica de Macaé de Cima. In Serra de Macaé de Cima: Diversidade Florística e Conservação em Mata Atlântica (H.C. Lima \& R.R. Guedes-Bruni, eds.). Jardim Botânico do Rio de Janeiro, Rio de Janeiro, p.75-87.

MORELLATO, L.P.C. \& LEITÃO FILHO, H.F. 1996. Reproductive phenology of climbers in a Southeasthern Brazilian forest. Biotropica 28:180-191.

MORELLATO, L.P.C. \& LEITÃO FILHO, H.F. 1998. Levantamento florístico da comunidade de trepadeiras de uma floresta semidecídua no Sudeste do Brasil. Boletim do Museu Nacional, nova série, Botânica 103:1-15.

NIMER, E. 1989. Climatologia do Brasil. Instituto Brasileiro de Geografia e Estatística, Rio de Janeiro.

OLIVEIRA FILHO, A.T. \& FONTES, M.A.L. 2000. Patterns of floristic differentiation among Atlantic forests in Southeastern Brazil and influence of climate. Biotropica 32:793-810.

PAGANO, S.N. \& LEITÃO FILHO, H.F. 1987. Composição florística do estrato arbóreo de mata mesófila semidecídua, no município de Rio Claro (Estado de São Paulo). Revista Brasileira de Botânica 10:37-48.

PAGANO, S.N., LEITÃO FILHO, H.F. \& SHEPHERD, G.J. 1987. Estudo fitossociológico em mata mesófila semidecídua no município de Rio Claro (Estado de São Paulo). Revista Brasileira de Botânica 10:49-62.

PAGANO, S.N., LEITÃO FILHO, H.F. \& CAVASSAN, O. 1995. Variação temporal da composição florística e estrtutura fitossociológica de uma floresta mesófila semidecídua Rio Claro - Estado de São Paulo. Revista Brasileira de Biologia 55:241-258.
PEÑALOSA, J. 1984. Basal branching and vegetative spread in two tropical rainforest lianas. Biotropica 16:1-9.

POLHILL, R.M. \& RAVEN, P.H. (eds.). 1981. Advances in legume systematics. Part 1. Royal Botanical Gardens, Kew.

PRANCE, F.E. 1994. A comparison of the efficacy of higher taxa and species numbers in the assessment of biodiversity in the neotropics. Philosophical Transactions of the Royal Society of London, Biological Sciences 345:89-99.

PUTZ, F.E. 1984. The natural history of lianas on Barro Colorado Island, Panama. Journal of Ecology 65:1713-1724.

PUTZ, F.E. \& CHAI, P. 1987. Ecological studies of lianas in Lambir National Park, Sarawak. Journal of Ecology 75:523-531.

PUTZ, F.E. \& WINDSOR, D.M. 1987. Liana phenology on Barro Colorado Island, Panama. Biotropica 19:334-341.

REZENDE, A.A. 1997. Levantamento florístico das espécies de lianas da Estação Ecológica do Noroeste Paulista São José do Rio Preto/Mirassol, SP, chave de identificação e diagnoses. Dissertação de mestrado, Universidade Estadual de Campinas, Campinas.

RIBEIRO, J.E.L.S., HOPKINS, M.J.G., VICENTINE, A., SOTHERS, C.A., COSTA, M.A.S., BRITO, J.M., SOUZA, M.A.D., MARTINS, L.H.P., LOHMANN, L.G., ASSUNÇÃO, P.A.C.L., PEREIRA, E.C., SILVA, C.F., MESQUITA, M.R. \& PROCÓPIO, L.C. 1999. Flora da Reserva Ducke: guia de identificação das plantas vasculares de uma floresta de terra firme na Amazônia Central. Inpa, Manaus.

RICHARD, P.W. 1952. The tropical rain forest: an ecological study. Cambridge University Press, Cambridge.

RIZZINI, C.T. 1963. Nota prévia sobre a divisão fitogeográfica (florístico-sociológica) do Brasil. Revista Brasileira de Geografia 25:3-84.

SALIS, S.M., SHEPHERD, G.J. \& JOLY, C.A. 1995. Floristic comparison of mesophytic semideciduous forests of the interior of the state of São Paulo, Southeast Brazil. Vegetatio 119:155-164.

TORRES, R.B., MARTINS, F.R. \& KINOSHITA, L.S. 1997. Climate, soil and tree flora relationships in forests in the state of São Paulo, southeastern Brazil. Revista Brasileira de Botânica 20:41-50.

VELOSO, H.P. 1991. Sistema fitogeográfico. In Manual Técnico da Vegetação Brasileira. Manuais Técnicos em Geociências 1. Fundação Instituto Brasileiro de Geografia e Estatística, Rio de Janeiro, p.9-38. 